\title{
An Airborne Target Localization Algorithm Based on UKF and Optimal Maneuver Strategy
}

\author{
Chunxiao Zhang ${ }^{1}$, Xiaofeng Shi ${ }^{1}$ \\ ${ }^{1}$ School of Electronics and Information Engineering, Beihang University Beijing, China
}

\begin{abstract}
The airborne bearing-only passive target localization performance is relevant with the specific filter and maneuver model. This paper presents a combination of Unscented Kalman Filtering (UKF) with control inputs and optimal route planning algorithm to improve the performance. Taking the minimum trace of unbiased UKF estimation covariance matrix as criteria, single-step heading traverse method is applied to reach every optimal solution. A single flight path simulation and Monte Carlo analysis validate that the optimal strategy can improve both the convergence and localization accuracy.
\end{abstract}

\section{Introduction}

Benefitting its flexibility and concealment, airborne single-observer localization is applied in interference investigation, radio rescue and military reconnaissance, etc. The bearing-only algorithm has been a long-term and most interesting topic in passive localization area [1]. Even now, in some certain circumstances, passive singlestation equipment can only detect the bearing of the target radiation source, which makes the bearing-only localization remain its significance [2-3].

Based on the airborne direction-finding system, a couple of emitter bearing angles relative to the aircraft, are collected at different points of the flight trajectory firstly. Then, the bearing-only algorithm estimates the emitter location by simple triangulation principle [4]. There are two problems in this estimation processing. At first, noises are inevitably introduced into the bearing measurements, which are propagated to final location estimation. Additionally, the mathematical model of bearing-based localization is non-linear. Filter is employed to solve the problems above, such as Extended Kalman Filtering (EKF) [5], Unscented Kalman Filtering (UKF) [6]. However, removing high-order parts in the Taylor series expansion, EKF suffers linearizing errors and degrades localization accuracy, even leads to the divergence[7]. Since no linearization is required, UKF is more suitable for states estimation in nonlinear systems, and attracts more attentions [8].

Another issue to be considered is that better observability should be maintained to ensure accurate bearing angles as possible. Besides the direction-finder equipment, the aircraft maneuver mode is critical for the angle measurement [9]. As a result, the localization accuracy and time are closely related to the specific aircraft maneuvering mode, which is investigated by lots of scholars already. The most applied, optimal criteria are Fisher Information Matrix (FIM) and its inverse matrix CRLB [10-14]. The posterior CRLB of the discrete-time nonlinear filtering problem to reduce the computational complexity was used in Ref.[15]. Steven C. Nardone proposed that the Hamilton-jacobi equation in the optimal control theory be used to find the optimal trajectory of the observation platform by maximizing the Fisher's information matrix lower bound[16]. Oshman and Davidson used differential inclusion to solve the resulting optimal control problem based on maximizing the determinant of the FIM[11]. In Ref. [17] and Ref.[18], adopting maximizing rate of change of the azimuth as the optimization rule, the optimization of single-observer trajectory of passive location was analyzed. In order to achieve the best estimation accuracy at each second of localization process, an adaptive observer maneuver control approach based on minimizing the GDOP(Geometrical Dilution of Precision) of target localization was presented in Ref.[19].

In this paper, the combination of an UKF filter and an optimal maneuver strategy is presented to improve convergence and accuracy of the airborne single-observer localization algorithm. In this strategy, taking the trace of covariance as the control criterion, UKF determines the optimal maneuvering path for better observability, resulting improved emitter localization.

\section{Mathematical model}

In general, the state and the measurement equations in the nonlinear filter model are

$$
\left\{\begin{array}{l}
X_{k+1}=\mathrm{f}\left(k, X_{k}\right)+w_{k} \\
Z_{k}=\mathrm{h}\left(k, X_{k}\right)+v_{k}
\end{array}\right.
$$


Where, the process noise $w_{k}$ is zero mean Gaussian white noise; the measurement noise $v_{k}$ is also zero mean Gaussian white noise, but independent with $w_{k}$.

As is shown in Fig. 1, source target $\mathrm{T}$ is located at $\left(\mathrm{x}_{T}, y_{T}\right)$.The aircraft moves from the origin as a straight motion with the velocity $u=\left[u_{x}, u_{y}\right]$ to measure the bearing of source target. $\phi_{k}$ is the target bearing of the aircraft detecting at the $\mathrm{k}^{\text {th }}$ observing points $\left(\mathrm{x}_{o k}, y_{o k}\right)$, the measurement equation for airborne single observer localization system is

$$
\begin{aligned}
\phi_{k} & =\arctan \left(\frac{y_{T}-y_{o k}}{x_{T}-x_{o k}}\right)+v_{k} \\
& =\arctan \frac{y_{k}}{x_{k}}+v_{k}
\end{aligned}
$$

Where, $\left[x_{k}, y_{k}\right]=\left[x_{T}-x_{o k}, y_{T}-y_{o k}\right]=X_{k}$ is relative position vector of airborne observer and target.

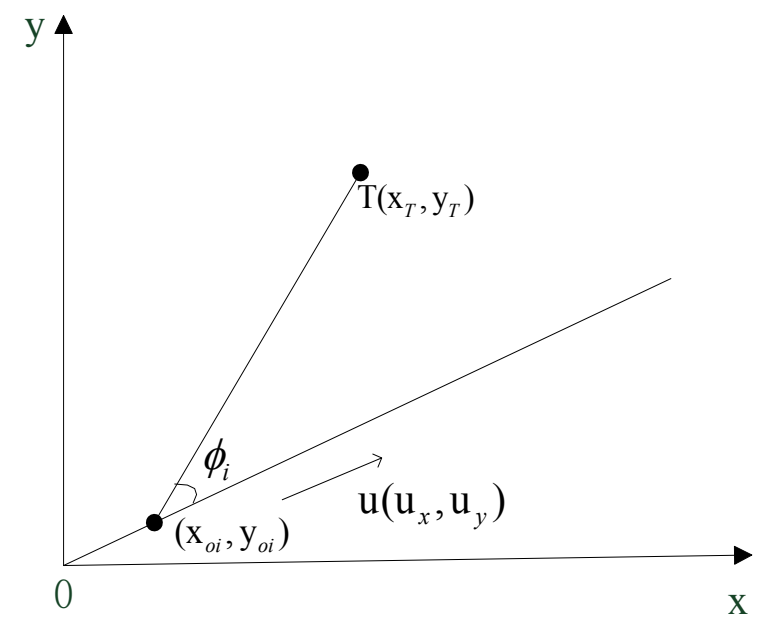

Figure 1. Airborne single observer passive of bearing-only method

The aircraft is moving fast and the measuring period is short, therefore slow moving or static targets can approximately be regarded as fixed. Taking aircraft speed $u$ as a control input, the system state equation is

$$
X_{k}=A X_{k-1}+B u_{k-1}+w_{k-1}
$$

Where, $A \in R^{n \times n}$ is coefficient matrix of state vector; $B \in R^{n \times l}$ is coefficient matrix of control input vector.

\section{Algorithm based on ukf with control inputs and optimal maneuver}

\subsection{UKF with control inputs}

The system equation of UKF with control inputs is

$$
\left\{\begin{array}{l}
X_{k+1}=A X_{k}+B u_{k}+w_{k} \\
\phi_{k}=h\left(k, X_{k}\right)+v_{k}
\end{array}\right.
$$

Where, $h\left(k, X_{k}\right)=\arctan \frac{y_{k}}{x_{k}}$.

The processing steps in UKF with control inputs are as follows:

1) Set initial state solution $\hat{X}_{0 \mid 0}$ and initial state error convariance matrix $P_{0 \mid 0}$ :

$$
\left\{\begin{array}{l}
\hat{X}_{0 \mid 0}=E\left[X_{0}\right] \\
P_{0 \mid 0}=E\left[\tilde{X}_{0 \mid 0} \tilde{X}_{0 \mid 0}^{T}\right]=E\left[\left(X_{0}-\hat{X}_{0 \mid 0}\right)\left(X_{0}-\hat{X}_{0 \mid 0}\right)^{T}\right]
\end{array}\right.
$$

2) $k \in[1, N]$ (iteration number)

(1) Calculate the sigma points $\chi_{i}(k \mid k)$

$$
\begin{aligned}
\chi_{i}(k \mid k) & =F\left(\hat{X}_{k \mid k}, P_{k \mid k}\right) \\
& =\left\{\begin{array}{lc}
\hat{X}_{k \mid k} & i=0 \\
\hat{X}_{k \mid k}+\left(\sqrt{(L+\lambda) P_{k \mid k}}\right) & i=1,2, \cdots, L \\
\hat{X}_{k \mid k}-\left(\sqrt{(L+\lambda) P_{k \mid k}}\right) & i=1+L, 2+L, \cdots, 2 L+1
\end{array}\right.
\end{aligned}
$$

Where, $\hat{X}_{k \mid k}$ is state solution at the $\mathrm{k}^{\text {th }}$ observing point, $P_{k \mid k}$ is state error convariance matrix solution, $\lambda=\alpha^{2}(L+\eta)-L$ is a scaling parameter. $\alpha$ is determines the spread of the sigma points around the mean state and is usually set to a small positive value. $\eta$ is a secondary scaling parameter (usually set to 0 ); $L$ is the dimension of the state vector.

(2) Update equations

$$
\begin{array}{r}
\chi_{i}(k+1 \mid k)=\mathrm{A} \chi_{i}(k \mid k)+\mathrm{B} u \\
\hat{X}_{k+1 \mid k}=\sum_{0}^{2 L} W_{i}^{(m)} \chi_{i}(k+1 \mid k) \\
P_{k+1 \mid k}=\sum_{i=0}^{2 L+1} W_{i}^{(c)} \Delta X_{i k+1 \mid k} \Delta X_{i k+1 \mid k}^{T}+Q_{k} \\
\xi_{i}(k+1, k)=h\left(k+1, \chi_{i}(k+1)\right) \\
\hat{\phi}_{k+1 \mid k}=\sum_{0}^{2 L} W_{i}^{(m)} \xi_{i}(k+1 \mid k) \\
P_{\phi \phi}=\sum_{i=0}^{2 L+1} W_{i}^{(c)} \Delta \Phi_{i k+1 \mid k} \Delta \Phi_{i k+1 \mid k}^{T}+R_{k} \\
P_{X \phi}=\sum_{i=0}^{2 L+1} W_{i}^{(c)} \Delta X_{i k+1 \mid k} \Delta \Phi_{i k+1 \mid k}^{T} \\
K_{k+1}=P_{X \phi} P_{X \phi}^{-1} \\
X_{k+1}=\hat{X}_{k+1 \mid k}+K_{k+1}\left(\phi_{k+1}-\hat{\phi}_{k+1 \mid k}\right) \\
P_{k+1 \mid k+1}=P_{k+1 \mid k}-K_{k+1} P_{\phi \phi} K_{k+1}^{T}
\end{array}
$$

Where,

$$
\Delta X_{i k+1 \mid k}=\chi_{i}(k+1 \mid k)-\hat{X}_{k+1 \mid k}
$$




$$
\begin{cases}W_{0}^{(m)}=\frac{\lambda}{\mathrm{L}+\lambda} & i=0 \\ W_{0}^{(m)}=\frac{\lambda}{\mathrm{L}+\lambda}+\left(1-\alpha^{2}+\beta\right) & i=0 \\ W_{i}^{(m)}=W_{i}^{(m)}=\frac{\lambda}{2(\mathrm{~L}+\lambda)} & i=1,2, \cdots, 2 \mathrm{~L}\end{cases}
$$

$\beta$ incorporates priori knowledge of distribution of the state (the optimal value is 2 ); $\xi_{i}(k+1, k), i=0,1,2, \cdots 2 \mathrm{~L}$ are the sigma points of the one-step predictive measurement $\phi_{k+1 \mid k} ; \Delta \Phi_{i k+1 \mid k}=\xi_{i}(k+1 \mid k)-\hat{\phi}_{k+1 \mid k}, Q_{k}$ is processing noise variance, $R_{k}$ is measurement noise variance.

\subsection{Optimal maneuver path planning}

The variance lower bound of the unbiased estimator is CRLB. As an unbiased estimation method, the mean square error of UKF with control inputs estimator is $P_{k \mid k}$. According to the statistical lemma [20],

$$
\begin{aligned}
P_{k \mid k} & =\operatorname{tr}\left(\operatorname{Cov}\left(\hat{X}_{k \mid k}\right)\right)+\left\|\mathrm{E} \hat{X}_{k \mid k}-X_{k}\right\|^{2} \\
& =\operatorname{tr} \operatorname{Cov}\left(\hat{X}_{k \mid k}\right)=\operatorname{tr}\left(P_{k \mid k}\right)
\end{aligned}
$$

Therefore, $\operatorname{tr}\left(P_{k \mid k}\right)$ can be used as optimal maneuvering optimization criteria. The $\operatorname{tr}(\bullet)$ represents the trace of the matrix; $\operatorname{Cov}\left(\hat{X}_{k \mid k}\right)$ is the covariance matrix. The problem discussed in this section is turn to solving the following equation:

$$
\left\{\begin{array}{l}
\min \operatorname{tr}\left(P_{k \mid k}\right)=\operatorname{tr}\left(P_{k \mid k-1}-K_{k} P_{\phi \phi} K_{k}^{T}\right) \\
\hat{X}_{k \mid k}=A \hat{X}_{k-1 \mid k-1}+B u_{k-1}
\end{array}\right.
$$

The solution of the equation (20) is actually a global optimization process. In order to make it easier to be solved and more practical for engineering, this paper presents a single-step optimal method. The method exhausts all the headings of the airborne observation platform at every step with the minimum $\operatorname{tr}\left(P_{k \mid k}\right)$ as the evaluation criterion, and obtains the optimal solution of each step. Finally, a local optimal solution is achieved.

The steps of one-step optimal control are described as follows:

1) At time $\mathrm{k}$, the relative position of the platform and target position $\hat{X}_{k \mid k}$ and $\hat{X}_{T}$ are estimated via UKF based on the last k-1 bearings of target and observation platform positions.

2) Traverse the $\mathrm{N}$ flight directions of the observation platform with a certain step length.

(1) In the flight direction $i(i \in[0, N-1])$ of the observation platform, use the UKF method to estimate possible position of observing platform and target source at next step and get the next possible state error convariance matrix $P_{k \mid k}$.

(2) Calculate $\operatorname{tr}\left(P_{k \mid k}\right)$.

(3) Repeat (1) and (2) until all possible flight directions are traversed, choose the direction with minimum $\operatorname{tr}\left(P_{k \mid k}\right)$ for the actual flight at next step.

\subsection{Algorithm steps}

Assuming the aircraft velocity flies with a constant speed and only the heading can be changed among $\pm 60^{\circ}$, the emitter position is estimated as follows:

1) Set initial value of the target source position and covariance.

2) Calculate all possible UKF predictions at time $\mathrm{k}$ and all predicted $\operatorname{tr}\left(P_{k \mid k}\right)$, find the direction with minimum $\operatorname{tr}\left(P_{k \mid k}\right)$ as the next direction of flight.

3) Update $u$, the control input of UKF. Where, $u=[|u| \cos (\theta),|u| \sin (\theta)], \theta$ is the angle between the direction of the aircraft and the $\mathrm{x}$-axis.

4) Repeat with 2), 3) until the end of positioning.

\section{Simulation and analysis}

Experiment One: Optimal path planning validation. The simulation environment is set as: the position of target source is $(100 \mathrm{~km}, 100 \mathrm{~km})$, the initial position of the aircraft is $(0 \mathrm{~km}, 0 \mathrm{~km})$, its velocity vector is $(200 \mathrm{~m} / \mathrm{s}, 0$ $\mathrm{m} / \mathrm{s})$. The observation interval is $1 \mathrm{sec}$; the bearing error is Gaussian white noise with the standard deviation of 1 degree. The heading change in each step in the optimal maneuvering strategy is 5 degrees, and the number of observations is 3000 times. The results of the trajectory simulation are shown in Fig. 2. Fig. 2 (a) shows the motion trajectory of the UKF with input controls and optimal maneuvering strategy. Fig. 2 (b) is the motion trajectory which does not take the optimal maneuvering strategy into account. The blue curve is the trajectory of the airborne observation platform. The "+" is the position estimation of the target and the red "O" is the target real position.

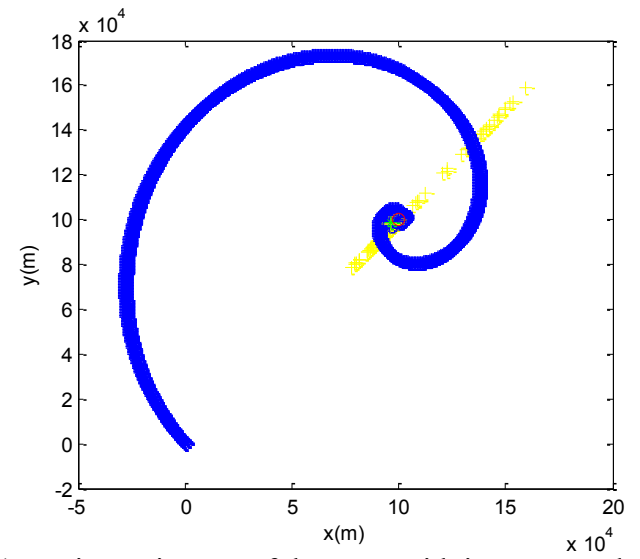

(a) Motion trajectory of the UKF with input controls and optimal maneuvering strategy 


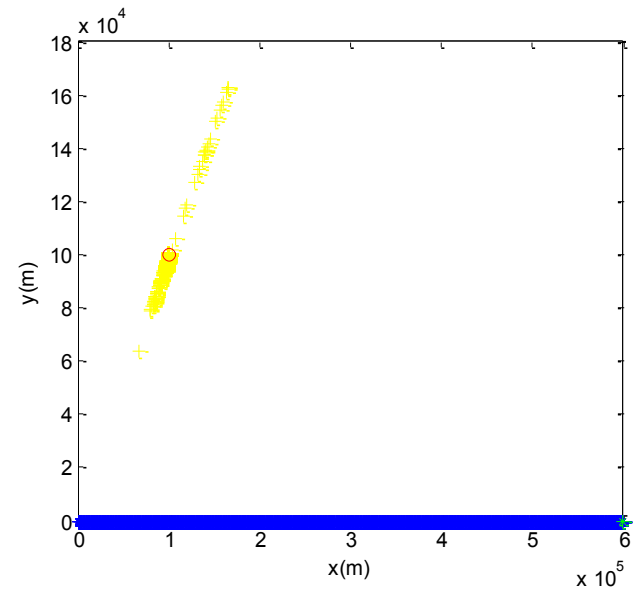

(b) Motion trajectory without the optimal maneuvering strategy.

Figure 2. Trajectories comparison

The comparison shows that under the influence of the optimal control algorithm, the trajectory of the observation platform is no longer a straight line, but a spiral motion. When the observation platform performs the optimal maneuver selection in each step, the heading of airborne platform has been changed. This results in changes of control inputs to ensure the observation platform observability and achieves the localization error convergence.

Experiment Two: Comparison of algorithms performance. The simulation parameters are same as Experiment One, except the number of observations is set 200. The number of Monte Carlo experiments is set to 50 . Fig. 3 shows the simulation results of the UKF algorithm both with and with no the optimal maneuver. The abscissa represents the number of observation while the ordinate indicates the error. In this paper, the relative localization error is used as the evaluation criterion of the algorithm precision, and the convergence threshold is set as $5 \%$. The relative error is defined as

$$
r=\frac{\left\|\hat{X}_{k \mid k}-X_{k}\right\|}{\left\|X_{T}-X_{0}\right\|} \times 100 \%
$$

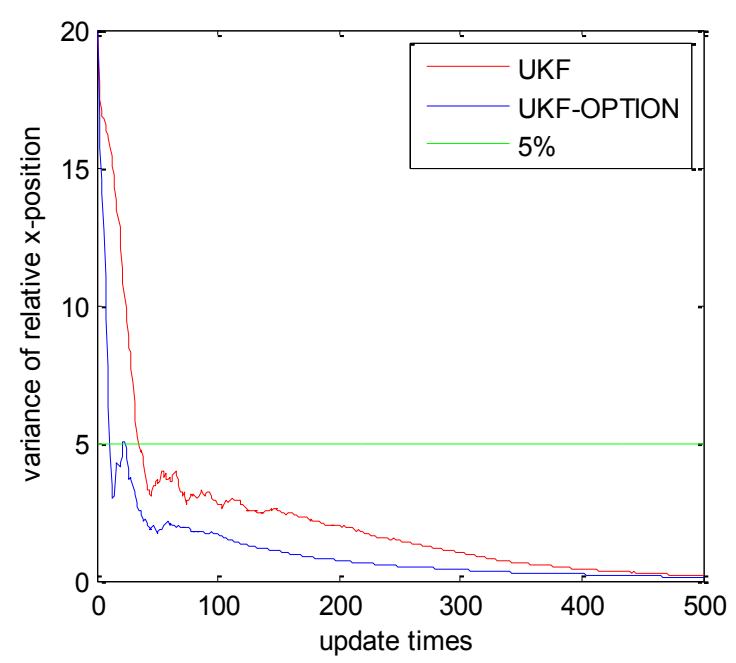

(a) Relative error of $\mathrm{x}$-coordinate

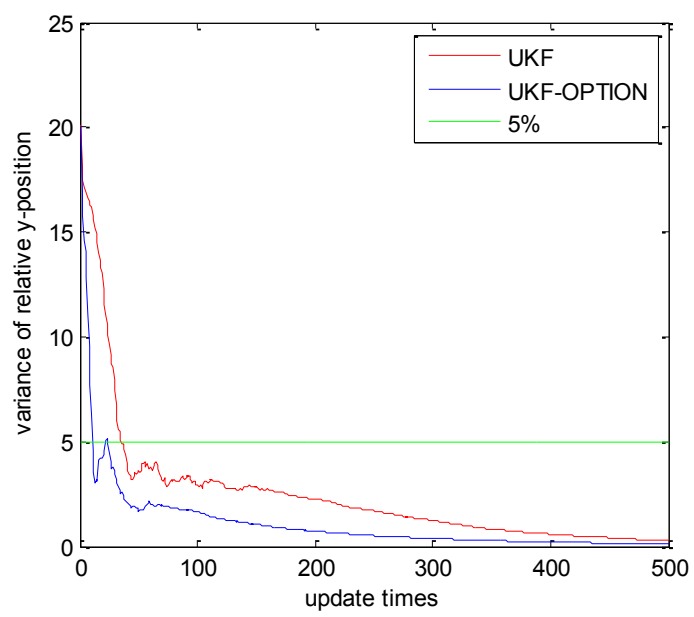

(b) Relative error of $y$-coordinate

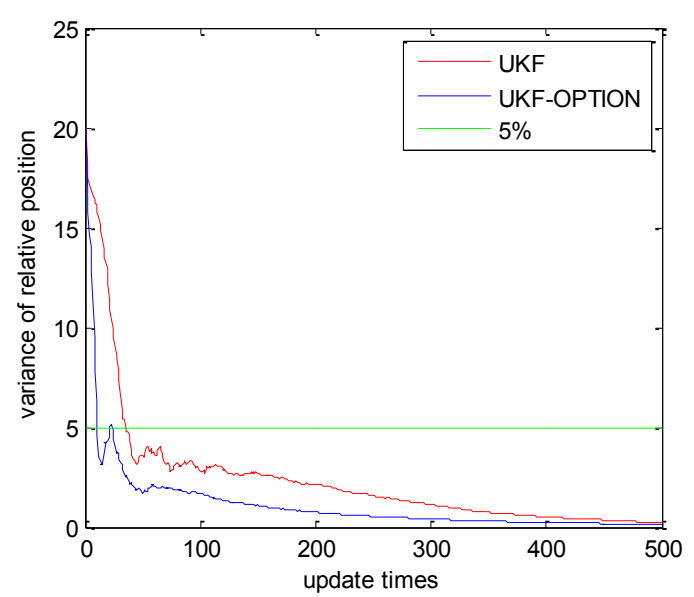

(c) Relative mean square error

Figure 3. Algorithm performance comparison of the UKF with and with no optimal maneuver

From the comparison among (a), (b) and (c) in Fig. 3, it can be concluded that with the same iteration times, the error of UKF algorithm with the optimal maneuver is smaller than the UKF algorithm only. It proves that the optimal maneuvering algorithm improves the localization accuracy. Moreover, after processing with the algorithm base on UKF and optimal maneuver, the relative error curve becomes smoother and can reach below the convergence threshold $(5 \%)$ in less iterations. It demonstrates that the optimal maneuver improves both the stability and convergence of the UKF localization algorithm.

\section{Conclusion}

The UKF algorithm with control inputs is suitable for solving the problem in bearing-only airborne passive single observer localization solution. In the UKF method, the control input has a certain influence on the convergence speed and localization accuracy which can be improved by optimizing control inputs. In this paper, a single station localization algorithm based on UKF with optimal inputs is proposed, where the minimum trace of state estimation covariance is taken as the criterion to 
optimize the maneuvering direction. The simulation experiments verify that the algorithm improves both the stability and convergence. The paper has built the bearings-only mathematic model under the condition that the target is fixed or moving slowly. If the target is also maneuvering, the optimal maneuvering of the observer should be further studied. Simultaneously, the calculation cost of the UKF algorithm is large. Other filtering methods to reduce the computational load remain to be further studied, too.

\section{References}

1. Y. Shan, Z. Sun, K. Huang. Asrpspace Electronics Warfare,6,4 (2001)

2. O. N. Gharehshiran,V. Krishamurthy.IEEE T SIGNAL PROCES,58(2010).

3. S. Chen, H. Wu, H. Zhang. Computer Science \& Service System, ( Nanjing, China,2012).

4. W. Su, G.Wang,P. Zhong, J. Xiu. Journal of Naval Aeronautical and Astronautical University,24(2009)

5. M.J. Moorman, T.E. Bullock. Aerospace and Electronics Conference (NAECON ,1992)

6. S. Julier, J. Uhlmann, H. F. Durrant-Whyte. IEEE T AUTOMAT CONTR, 45(2000).

7. N. J. Gordon, D.J Salmond, A.F.M. Smith. IEE Proceedings-F, 140(1993)

8. C. Liu. Passive location and tracking (Xidian University Press,Xian,China,2011)
9. Z. Xu, A. Sheng, Z. Guo.Proceedings of IEEE International Conference on Networking, Sensing and Control (Sanya, China, 2008).

10. P. T. Liu. An optimum approach in target tracking with bearings measurements.Journal of Optimization Theory and Applications, 1987, 56(2): 205 - 214.

11. Y. Oshman, P. Davidson. IEEE T AERO ELEC SYS, 35(1999)

12. J M Passerieux, D Van Cappel. IEEE T AERO ELEC SYS, 34(1998)

13. J P Helferty,D R Mudgett. Proceedings of the 32nd Conference on Decision and Control (San Antonlo, Texas, 1993).

14. W Zhang, M Liu, Z Wang etal. Second International Conference on Information and Computing Science,(2009)

15. P.Tichavsky, C. H. Muravchik, A. Nehorai. IEEE T SIGNAL PROCES, 46(1998)

16. S. C. Nardone, V. J. Aidala. IEEE T AERO ELEC SYS,17 ( 1981)

17. Y Zhang. Fire Control Radar Technology, 33, 5 (2004)

18. Z Dai, X Deng. Aerospace Electronic Warfare, 4 (2007).

19. X Deng, Y Zhou, Q Lu. Acta Electronica Sinica,4 (2007)

20. G Wang, M Chen, L Chen.Linear Statistical Model (Higher Education Press, Beijing China,1999). 